\title{
AN ANALYTICAL THEORY FOR A GYROSTATIC EARTH
}

\author{
R. MOLINA AND A. VIGUERAS \\ Depto. de Matemática Aplicada y Estadistica, Univ. Murcia \\ Cartagena, Spain
}

\begin{abstract}
In this paper, we consider the problem of the rotation of the Earth, using a stationary triaxial gyrostat as a model. The problem is formulated by means of dimensionless canonical variables of Serret-Andoyer, referred to the mean ecliptic of date, in a similar way to Kinoshita (1977). We choose the constant components of the gyrostatic momentum in such a way that the period of the polar motion corresponds to Chandler's period. Finally, the problem is integrated by means of Deprit's perturbation method.
\end{abstract}

\section{Introduction}

The model of a rigid body to represent celestial bodies when studying their motion implies the absence of internal or relative motions. This is not always suitable as was shown by Volterra (1899) in the study of the variation of latitude on the Earth's surface, explaining then the anomalies of the free rotation by means of internal or relative motions which do not modify the distribution of masses (subterranean currents, atmospheric currents, etc.).

The theory of rotation of the Earth about its center of mass constructed by Woolard (1953) and adopted by the IAU as the international standard, considered that, dynamically, the Earth is a symmetrical rigid body.

By using the Serret-Andoyer canonical variables (Andoyer, 1923), and a moving plane of reference (the mean ecliptic of date), Kinoshita (1977) developed a theory of the Earth's rotation. He adopted a triaxial rigid Earth model and Hori's perturbation method. This theory has two fundamental advantages: 1) it treats separately the motions of the rotation axis and of the angular momentum axis, and 2) it utilizes the mean ecliptic of date as plane of reference. In this way, mixed secular terms do not appear in the perturbation function.

I. M. Wytrzyszczak, J. H. Lieske and R. A. Feldman (eds.), Dynamics and Astrometry of Natural and Artificial Celestial Bodies, 307, 1997.

(C) 1997 Kluwer Academic Publishers. Printed in the Netherlands. 
R. Cid and A. Vigueras (1990), studied the problem of the Earth's rotation, when it is attracted by the Sun and the Moon, using as a model a stationary symmetric gyrostat, and they utilized the canonical variables $\pi, \zeta, \nu, P_{\pi}, P_{\zeta}, P_{\nu}$, introduced by Cid and Correas (1973) also referred to the mean ecliptic of date, and Deprit's perturbation method (1969).

In the present paper, we use as a model of the Earth a stationary triaxial gyrostat which has the two first components of the gyrostatic momentum identical to zero and the third one constant, in such a way that in the absence of external forces the free solution describes the Chandler period. We propose an intermediate model between the one of Kinoshita and that of Getino and Ferrándiz (1991). The problem has been formulated in terms of a set of dimensionless variables $\pi, \zeta, \nu, P_{\pi}, P_{\zeta}, P_{\nu}$, referred to the mean ecliptic of date, which is determined by the planetary presence. In addition, to simplify, we suppose that the Sun moves in a Keplerian orbit with null inclination and the Moon moves in a Keplerian orbit whose nodal and inclination arguments are constant with respect to the mean ecliptic of date.

\section{Statement of the Problem}

Let us suppose the Earth to be a gyrostat with constant gyrostatic momentum and which turns around an axis passing through its center of mass $O$, with instantaneous angular velocity $\vec{\omega}$. Therefore, we can consider a system of the principal axes of inertia $O x y z$, which are rigidly attached to the rigid part of the Earth, whose principal moments of inertia are denoted by $A, B, C$.

Let us consider a system $O X Y Z$, whose axes are parallel to those of other inertial ones, and whose $O X Y$ plane corresponds to the mean ecliptic of the initial epoch $t_{0}=0$. The mean ecliptic for the epoch $t$ (ecliptic of date), given by the $O X^{*} Y^{*}$ plane of a new system $O X^{*} Y^{*} Z^{*}$, is referred to the $O X Y Z$ system through the functions $\Omega(t)$, and $I(t)$ (nodal and inclination angles), which are given by the following expressions (Newcomb, 1906)

$$
\sin I \sin \Omega=\sum_{i=1}^{\infty} F_{i} t^{i}, \quad \sin I \cos \Omega=\sum_{i=1}^{\infty} G_{i} t^{i}
$$

where the coefficients $F_{i}, G_{i}$, are constants.

In addition, we suppose that the Earth is attracted according to Newton's law by two material points (the Moon and the Sun) of masses $M$, $M^{\prime}$, respectively, and that such masses describe elliptical orbits around $O$, in such a way that the Sun moves into the $O X^{*} Y^{*}$ plane and the Moon in a plane, whose nodal and inclination angles with respect to the $O X^{*} Y^{*}$ plane are $h$ and $j$, respectively. 
If we use the $O X Y$ plane as a reference, the variables $\pi, \zeta, \nu, P_{\pi}, P_{\zeta}, P_{\nu}$, are suitable to study the Earth rotation (Cid and Vigueras, 1990). Let us carry out the transformation

$$
\left(P_{\pi}^{\prime}, P_{\zeta}^{\prime}, P_{\nu}^{\prime}\right)=\frac{1}{A w_{0}}\left(P_{\pi}, P_{\zeta}, P_{\nu}\right), \quad t^{\prime}=t w_{0}
$$

to other dimensionless variables, being $w_{0} \neq 0$, the initial angular velocity of the Earth. Then, we obtain for the Hamiltonian (where we have suppressed the prime symbols for the new variables) the expression

$$
\mathcal{H}=\mathcal{H}_{T}+R+U+U^{\prime}
$$

where

$$
\begin{aligned}
\mathcal{H}_{T}= & \frac{1}{2}\left[\frac{A+B}{2 B} P_{\zeta}^{2}+\left(\frac{A}{C}-\frac{A+B}{2 B}\right) P_{\nu}^{2}\right]+\frac{A-B}{4 B}\left(P_{\zeta}^{2}-P_{\nu}^{2}\right) \cos 2 \nu \\
& -\sqrt{P_{\zeta}^{2}-P_{\nu}^{2}}\left(\frac{a_{1}}{A w_{0}} \sin \nu+\frac{a_{2}}{B w_{0}} \cos \nu\right)-\frac{a_{3}}{C w_{0}} P_{\nu} \\
R= & -P_{\pi} \frac{\dot{\Omega}}{w_{0}} \cos I+\sqrt{P_{\zeta}^{2}-P_{\pi}^{2}}\left(\frac{\dot{\Omega}}{w_{0}} \sin I \cos \pi-\frac{\dot{I}}{w_{0}} \sin \pi\right) \\
U= & \frac{3 G M}{2 r^{3} A w_{0}^{2}}\left((B-A) \beta^{2}+(C-A) \gamma^{2}\right) \\
U^{\prime}= & \frac{3 G M^{\prime}}{2 r^{\prime 3} A w_{0}^{2}}\left((B-A) \beta^{2}+(C-A) \gamma^{\prime 2}\right)
\end{aligned}
$$

being $(\beta, \gamma),\left(\beta^{\prime}, \gamma^{\prime}\right)$ the second and third direction cosines of the position vectors Earth-Moon and Earth-Sun, respectively, with respect to the system of the principal axes of inertia Oxyz. In this statement the terms of the lunisolar potential of power superior to $r^{-3}$ and $r^{\prime-3}$ are omitted, by assuming that these terms are sufficiently small.

The functions $\left(\beta^{2}, \gamma^{2}\right)$ and $\left(\beta^{2}, \gamma^{2}\right)$ can be expressed in the canonical variables by means of the equalities

$$
\begin{aligned}
\beta^{2} & =\sum_{i, s, p, q} \beta_{i, s, p, q} \cos (i \zeta+s(\pi-h)+p \nu+q \omega) \\
\gamma^{2} & =\sum_{i, s, q} \gamma_{i, s, q} \cos (i \zeta+s(\pi-h)+q \omega) \\
\beta^{\prime 2} & =\sum_{i, p, q} \beta_{i, p, q}^{\prime} \cos \left(i \zeta+p \nu+q\left(\omega^{\prime}-\pi\right)\right) \\
\gamma^{\prime 2} & =\sum_{i, q} \gamma_{i, q}^{\prime} \cos \left(i \zeta+q\left(\omega^{\prime}-\pi\right)\right)
\end{aligned}
$$


where $i \in\{0,1,2\}, s \in\{-2,-1,0,1,2\}, p, q \in\{-2,0,2\}$ and the coefficients $\beta_{i, s, p, q}, \gamma_{i, s, q}, \beta_{i, p, q}^{\prime}, \gamma_{i, q}^{\prime}$, are functions of the moments $P_{\pi}, P_{\zeta}, P_{\nu}$, given in Molina (1996). The variables $\omega=\omega_{0}+f, \omega^{\prime}=\omega_{0}^{\prime}+f^{\prime}$, define the positions of the Moon and the Sun by means of the true anomalies $f, f^{\prime}$, and the perigee's arguments $\omega_{0}, \omega_{0}^{\prime}$.

\section{The Study of the Different Terms of the Hamiltonian}

In order to separate $\mathcal{H}$ in different perturbation orders, we have considered the equalities

$$
\begin{array}{rlrl}
\frac{3 G M}{2 r^{3}} \frac{B-A}{A w_{0}^{2}} & =\frac{1}{2} e_{0}\left(\frac{a}{r}\right)^{3}, & \frac{3 G M}{2 r^{3}} \frac{C-A}{A w_{0}^{2}}=\frac{1}{2} e_{1}\left(\frac{a}{r}\right)^{3} \\
\frac{3 G M^{\prime}}{2 r^{\prime 3}} \frac{B-A}{A w_{0}^{2}}=\frac{1}{2} e_{0}^{\prime}\left(\frac{a^{\prime}}{r^{\prime}}\right)^{3}, & \frac{3 G M^{\prime}}{2 r^{\prime 3}} \frac{C-A}{A w_{0}^{2}}=\frac{1}{2} e_{1}^{\prime}\left(\frac{a^{\prime}}{r^{\prime}}\right)^{3}
\end{array}
$$

with

$$
\begin{array}{lll}
e_{0}=3\left(\frac{n}{w_{0}}\right)^{2} \frac{M}{M+M_{T}} \frac{B-A}{A}, & e_{1}=\left(\frac{n}{w_{0}}\right)^{2} \frac{M}{M+M_{T}} \frac{C-A}{A} \\
e_{0}^{\prime}=3\left(\frac{n^{\prime}}{w_{0}}\right)^{2} \frac{M^{\prime}}{M^{\prime}+M_{T}} \frac{B-A}{A}, & e_{1}^{\prime}=\left(\frac{n^{\prime}}{w_{0}}\right)^{2} \frac{M^{\prime}}{M^{\prime}+M_{T}} \frac{C-A}{A}
\end{array}
$$

where $n, n^{\prime}$ are the mean motions of the Moon and the Sun, respectively, $M_{T}$ is the mass of the Earth and we take $w_{0}=2 \pi \mathrm{rad} / \mathrm{sid}$.day.

By the relations (Kinoshita, 1990; Fukushima, 1995)

$$
\begin{aligned}
M_{T}=81.3 M, & M^{\prime}=332.958 M_{T} \\
n=\frac{2 \pi}{27.396} \mathrm{rad} / \mathrm{sid} . \mathrm{day}, & n^{\prime}=\frac{2 \pi}{366.25} \mathrm{rad} / \mathrm{sid} . \mathrm{day} \\
\frac{2 C-A-B}{2 C}=3.2739935 \cdot 10^{-3}, & \frac{(C-A)(C-B)}{A B}=1.0758 \cdot 10^{-5}
\end{aligned}
$$

we obtain

$$
\begin{gathered}
e_{0}=1.10102 \cdot 10^{-9}, \quad e_{1}=1.5983 \cdot 10^{-7} \\
e_{0}^{\prime}=5.07009 \cdot 10^{-10}, \quad e_{1}^{\prime}=7.36025 \cdot 10^{-8} \\
U+U^{\prime}=\frac{e_{0}^{\prime}}{2}\left(\delta\left(\frac{a}{r}\right)^{3} \beta^{2}+\left(\frac{a^{\prime}}{r^{\prime}}\right)^{3} \beta^{\prime 2}\right)+\frac{e_{1}^{\prime}}{2}\left(\delta_{1}\left(\frac{a}{r}\right)^{3} \gamma^{2}+\left(\frac{a^{\prime}}{r^{\prime}}\right)^{3} \gamma^{\prime 2}\right)
\end{gathered}
$$

where we have defined $\delta=e_{0} / e_{0}^{\prime}=2.171598, \delta_{1}=e_{1} / e_{1}^{\prime}=2.171529$. 
The terms of the complementary function $R$ can be written (Vigueras, 1983) in the form

$$
\begin{aligned}
R= & P_{\pi}\left(0.23602 \cdot 10^{-7}+0.58027 \cdot 10^{-9} t\right)-\left[0.53943 \cdot 10^{-11} t \cos \pi+\right. \\
& \left.+\left(0.99319 \cdot 10^{-9}-0.22646 \cdot 10^{-10} t\right) \sin \pi\right] P_{\zeta} S_{\pi}
\end{aligned}
$$

where $t$ is expressed in tropic centuries measured from 1850 .

If we suppose that the two first components of the gyrostatic momentum are null, $a_{1}=a_{2}=0$, the third constant component can be chosen in such a way that the free polar motion has a period of 430 days (Chandler's period). So, the solution of the equations of free motion of a triaxial gyrostat, if we suppose that $w_{3} \simeq w_{0}=2 \pi \mathrm{rad} / \mathrm{sid}$. day, can be approximated in the form

$$
w_{1}=a_{0} \cos \left(\sqrt{\rho \tau} t+b_{0}\right), \quad w_{2}=\sqrt{\frac{\tau}{\rho}} a_{0} \sin \left(\sqrt{\rho \tau} t+b_{0}\right)
$$

being $\rho=-\frac{B-C}{A} w_{0}+\frac{a_{3}}{A}, \tau=\frac{C-A}{B} w_{0}+\frac{a_{3}}{B}$, and $a_{0}, b_{0}$ are constant. This solution corresponds to an elliptic motion of the pole with a period $T=$ $2 \pi / \sqrt{\rho \tau}$, and making $T=430$, we obtain $b_{3}=a_{3} / C w_{0}=-0.95169 \cdot 10^{-3}$.

Then, taking the above hypotheses into account, and introducing the constants $c_{0}=\frac{A+B}{2 B}, c_{1}=\frac{A}{C}-c_{0}, c_{2}=\frac{A-B}{4 B}$, the Hamiltonian (3) can be written in the form

$$
\begin{aligned}
\mathcal{H}= & \frac{1}{2}\left(c_{0} P_{\zeta}^{2}+c_{1} P_{\nu}^{2}\right)+c_{2}\left(P_{\zeta}^{2}-P_{\nu}^{2}\right) \cos 2 \nu-b_{3} P_{\nu}+R+ \\
& +\frac{1}{2} e_{0}^{\prime}\left(\delta\left(\frac{a}{r}\right)^{3} \beta^{2}+\left(\frac{a^{\prime}}{r^{\prime}}\right)^{3} \beta^{\prime 2}\right)+\frac{1}{2} e_{1}^{\prime}\left(\delta^{\prime}\left(\frac{a}{r}\right)^{3} \gamma^{2}+\left(\frac{a^{\prime}}{r^{\prime}}\right)^{3} \gamma^{\prime 2}\right)
\end{aligned}
$$

Now, for the integration by means of a perturbation method, the Hamiltonian (8) can be written as follows

$$
\mathcal{H}=\mathcal{H}_{0}+\mathcal{H}_{1}
$$

being

$$
\mathcal{H}_{0}=\frac{1}{2}\left(c_{0} P_{\zeta}^{2}+c_{1} P_{\nu}^{2}\right)-b_{3} P_{\nu}, \quad \mathcal{H}_{1}=\mathcal{H}-\mathcal{H}_{0}
$$

\section{Hamiltonian of the Secular Motion}

The expressions (9), (10) and (4) put that $\mathcal{H}$ depends on variables $(\pi, \zeta, \nu)$ and the time $t$, establishing this dependence by means of Hansen's developments (Tisserand, 1889) for the elliptic motion. 
The unperturbed motion of Hamiltonian $\mathcal{H}_{0}$ is easily integrable because it has not angular variables, and its solution is given by

$$
\begin{aligned}
\pi & =\pi_{0}, & \zeta & =c_{0} P_{\zeta}^{0} t+\zeta_{0}, & \nu & =\left(c_{1} P_{\nu}^{0}-b_{3}\right) t+\nu_{0} \\
P_{\pi} & =P_{\pi}^{0}, & P_{\zeta} & =P_{\zeta}^{0}, & P_{\nu} & =P_{\nu}^{0}
\end{aligned}
$$

where $\pi_{0}, \zeta_{0}, \nu_{0}, P_{\pi}^{0}, P_{\zeta}^{0}, P_{\nu}^{0}$ are constants of integration.

In this way, the problem can be integrated by means of a perturbation method. We shall use the homogeneous formalism and Deprit's method to eliminate, in the absence of resonances, the periodic terms and obtain the Hamiltonian of the secular motion in the form

$$
\mathcal{H}^{\prime \prime \prime}\left(P_{\pi}^{\prime \prime \prime}, P_{\zeta}^{\prime \prime \prime}, P_{\nu}^{\prime \prime \prime}\right)=\mathcal{H}_{0}^{\prime \prime \prime}\left(P_{\zeta}^{\prime \prime \prime}, P_{\nu}^{\prime \prime \prime}\right)+\mathcal{H}_{1}^{\prime \prime \prime}\left(P_{\pi}^{\prime \prime \prime}, P_{\zeta}^{\prime \prime \prime}, P_{\nu}^{\prime \prime \prime}\right)
$$

Then, by integrating the equations of motion corresponding to the above Hamiltonian, we obtain the secular perturbations. All necessary equations and consequences will be given in Molina (1996).

\section{References}

Andoyer, H.: 1923, Cours de Mécanique Céleste, Gauthier-Villar, Paris.

Cid, R. and Correas, J.M.: 1973, Act. I Jornadas Matem. Hispano-Lusas, 439-452.

Cid, R. and Vigueras, A.: 1990, Rev. Acad. Ciencias Zaragoza, 45, 83-93.

Deprit, A.: 1969, "Canonical transformations depending on a small parameter", Celest. Mech. 1, 12-30.

Fukushima, T.: 1995, "A numerical scheme to integrate the rotational motion of a rigid body", private communication.

Getino, J., and Ferrándiz, J.M.: 1991, "A Hamiltonian theory for an elastic Earth: First order analytical integration", Celest. Mech. \& Dyn. Astron. 51, 35-65.

Kinoshita, H.: 1977, "Theory of the rotation of the rigid Earth", Celest. Mech. 15, $277-326$.

Kinoshita, H. and Souchay, J.: 1990, "The theory of the nutation for the rigid Earth model at the second order", Celest. Mech. \& Dyn. Astron. 48, 187-265.

Molina, R.: 1996, Ph.D. thesis (in preparation), Univ. de Murcia, Spain.

Vigueras, A.: 1983, Movimiento Rotatorio de Giróstatos y Aplicaciones, Ph.D. Thesis, Univ. de Zaragoza, Spain.

Volterra, V.: 1899, Acta Mathematica 22, 201. 